\title{
Periodic flow due to non-torsional oscillations of eccentric rotating porous disks in the presence of a magnetic field
}

\author{
H. Volkan Ersoy \\ Department of Mechanical Engineering, Yildiz Technical University, Istanbul, Turkey, E-mail: hversoy@yildiz.edu.tr \\ cross'ref http://dx.doi.org/10.5755/j01.mech.23.3.14101
}

\section{Introduction}

The study of flow between eccentric rotating disks has attracted interest of many investigators. Maxwell and Chartoff [1] pointed out that it is possible to determine the complex dynamic viscosity of an elastico-viscous liquid by means of eccentric rotating disks. An exact solution for the flow of a Newtonian fluid between eccentric rotating disks was first obtained by Abbott and Walters [2]. In the case of a viscoelastic fluid, they also found a solution with the help of a perturbation method. Rajagopal [3] showed that this motion is one with constant principal relative stretch history. A list of references dealing with the flow between eccentric rotating disks can be found in [4-5].

Time-dependent flows due to non-torsional oscillations of eccentric disks rotating with the same angular velocity in the case of a Newtonian fluid have also received attention. Erdoğan [6] considered that the disks start to rotate eccentrically and the lower disk executes non-torsional oscillation while they are initially rotating about a common axis. Later, Erdoğan [7] took into account that the disks start to rotate eccentrically and both the disks execute non-torsional oscillations in the same direction while they are initially rotating about a common axis. Ersoy [8] examined the unsteady symmetrical flow due to the non-torsional oscillations of the disks in their own planes and in the opposite directions while they are initially rotating eccentrically. Recently, Ersoy [9] studied the periodic flow produced by nontorsional oscillations of two porous disks in their own planes and in the opposite directions while they are initially rotating about non-coincident axes.

This paper investigates the periodic flow due to the non-torsional oscillations in their own planes and in the opposite directions of the porous disks while they are initially rotating with the same angular velocity about two non-coincident axes under the effect of a uniform magnetic field. An analytical solution in the complex form for the dimensionless shear stresses in the fluid is presented in terms of the Hartmann number, the suction/injection velocity parameter, the Reynolds number, the dimensionless velocity amplitude of oscillation in the $x$ - and $y$-directions, the ratio of the frequency of oscillation to the angular velocity of the disks, and the dimensionless time. The main aim of this paper is to study the effect of the magnetic field on the horizontal force applied by the fluid on the disks. Thus, the analysis in [9] is extended to the flow in the presence of a magnetic field. When the frequency of oscillation is less than, equal to, and larger than the angular velocity of the disks, the effect of the Hartmann number on the shear stresses corresponding to the components of the horizontal force is examined through the graphs.

\section{Basic equations and solution}

This paper is concerned with the periodic flow induced by non-torsional oscillations of eccentric rotating porous disks under the influence of a uniform magnetic field. The two insulated porous disks are initially rotating with the same angular velocity about two parallel axes perpendicular to the disks while a magnetic field is present. The direction of constant axial velocity is upward. The disks suddenly start to perform non-torsional oscillations in their own planes and in the opposite directions. When the oscillation motion is set up, the flow contains transients for small times and then the motion starts to be periodic in time.

Let us consider an incompressible Newtonian fluid between eccentric rotating porous disks. The top and bottom disks located at $z= \pm h$ are initially rotating about the $z^{\prime}$ and $z^{\prime \prime}$ - axes with the same angular velocity $\Omega$, respectively. The distance between the axes of rotation is $2 \ell$ and the eccentricity is formed along the $y$-axis. The constant axial velocity is in the positive $z$ - direction. Thus, the top disk is subjected to a uniform suction whereas there is a uniform injection at the bottom disk. A uniform magnetic induction $\boldsymbol{B}_{0}$ is applied to the insulated disks in the positive $z$ direction. The induced magnetic field is neglected under the assumption of a small magnetic Reynolds number. The top and bottom disks start to execute non-torsional oscillations in their own planes with the velocities $\boldsymbol{U}$ and $-\boldsymbol{U}$, respectively, where $\boldsymbol{U}=\left(U_{x} \boldsymbol{i}+U_{y} \boldsymbol{j}\right) \sin n t, \quad n$ is the frequency of the oscillation and $t$ is the time. After the initial transients disappear, the periodic flow occurred in the fluid is studied. The distance between the axes of rotation is fixed during the motion. The geometry of problem is shown in Fig. 1.

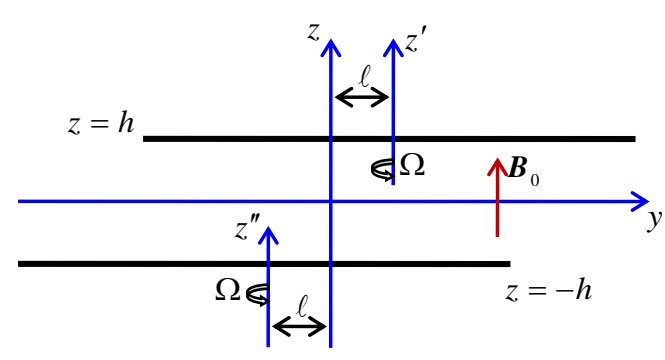

Fig. 1 Flow geometry

The equations governing the flow are:

$$
\rho \frac{D \boldsymbol{v}}{D t}=\nabla \cdot \boldsymbol{T}+\boldsymbol{J} \times \boldsymbol{B}
$$




$$
\nabla \cdot v=0
$$

where $\rho$ is the density, $D / D t$ the material time derivative, $\boldsymbol{v}=(u, v, w)$ the velocity vector, $\boldsymbol{T}$ the Cauchy stress tensor, $\boldsymbol{J}=\left(J_{x}, J_{y}, J_{z}\right)$ the current density, $\boldsymbol{B}$ the magnetic induction.

Therefore, the appropriate initial and boundary conditions for the velocity field can be written in the following form:

$u=-\Omega y+\hat{f}(z), \quad v=\Omega x+\hat{g}(z)$ at $t=0 ;$

$u=-\Omega(y-\ell)+U_{x} \sin n t, v=\Omega x+U_{y} \sin n t$ at $z=h ;$

$u=-\Omega(y+\ell)-U_{x} \sin n t, v=\Omega x-U_{y} \sin n t ;$

at $z=-h$;

$u=-\Omega y, \quad v=\Omega x \quad$ at $z=0$,

where the functions $\hat{f}(z)$ and $\hat{g}(z)$ represent the translational velocity components belonging to the steady flow of a Newtonian fluid between eccentric rotating porous disks in the presence of a magnetic field.

The initial and boundary conditions presented by Eqs. (3)-(6) suggest that the components of the velocity vector can be written as follows:

$$
u=-\Omega y+f(z, t), \quad v=\Omega x+g(z, t), w=w_{0},
$$

where the axial velocity is constant as a result of the incompressibility of the fluid. Inserting Eq. (7) into Eq. (1), we obtain:

$$
\begin{aligned}
& \frac{1}{\rho} \frac{\partial p}{\partial x}=v \frac{\partial^{2} f}{\partial z^{2}}-w_{0} \frac{\partial f}{\partial z}-\frac{\partial f}{\partial t}+\Omega(\Omega x+g)+\frac{B_{0} J_{y}}{\rho} ; \\
& \frac{1}{\rho} \frac{\partial p}{\partial y}=v \frac{\partial^{2} g}{\partial z^{2}}-w_{0} \frac{\partial g}{\partial z}-\frac{\partial g}{\partial t}+\Omega(\Omega y-f)-\frac{B_{0} J_{x}}{\rho} ; \\
& \frac{1}{\rho} \frac{\partial p}{\partial z}=0,
\end{aligned}
$$

where $p$ is the pressure, $v$ the kinematic viscosity of the fluid, $B_{0}$ the magnitude of the magnetic induction. Using $\boldsymbol{J}=\sigma(\boldsymbol{E}+\boldsymbol{v} \times \boldsymbol{B})$, we have:

$$
J_{x}=\sigma\left(E_{x}+v B_{0}\right), J_{y}=\sigma\left(E_{y}-u B_{0}\right), J_{z}=\sigma E_{z},
$$

where $\sigma$ is the electrical conductivity of the fluid and $\boldsymbol{E}=\left(E_{x}, E_{y}, E_{z}\right)$ is the electric field. Since the disks are insulated, we get $J_{z}=0$ and $E_{z}=0$.

$$
\begin{aligned}
& \text { Using } \nabla \times \boldsymbol{E}=-\frac{\partial \boldsymbol{B}}{\partial t}, \text { we obtain: } \\
& v \frac{\partial^{2} f}{\partial z^{2}}-w_{0} \frac{\partial f}{\partial z}-\frac{\partial f}{\partial t}+\Omega g-\frac{\sigma B_{0}^{2}}{\rho} f=C_{1}(t) ;
\end{aligned}
$$

$$
v \frac{\partial^{2} g}{\partial z^{2}}-w_{0} \frac{\partial g}{\partial z}-\frac{\partial g}{\partial t}-\Omega f-\frac{\sigma B_{0}^{2}}{\rho} g=C_{2}(t),
$$

where $C_{1}(t)$ and $C_{2}(t)$ are functions of time. Combining Eqs. (12)-(13), we obtain:

$$
\frac{\partial^{2} \bar{F}}{\partial \zeta^{2}}-\alpha \sqrt{R} \frac{\partial \bar{F}}{\partial \zeta}-R \frac{\partial \bar{F}}{\partial \tau}-\left(M^{2}+i R\right) \bar{F}=\bar{K}(\tau),
$$

with

$$
\begin{aligned}
& \bar{F}(\zeta, 0)=\frac{\hat{f}(z)+i \hat{g}(z)}{\Omega \ell} ; \\
& \bar{F}(1, \tau)=\left(1+V_{x} \sin k \tau\right)+i\left(V_{y} \sin k \tau\right) ; \\
& \bar{F}(-1, \tau)=-\left(1+V_{x} \sin k \tau\right)-i\left(V_{y} \sin k \tau\right) ; \\
& \bar{F}(0, \tau)=0,
\end{aligned}
$$

where

$$
\left.\begin{array}{l}
\bar{F}(\zeta, \tau)=\frac{f(z, t)+i g(z, t)}{\Omega \ell} ; \zeta=\frac{z}{h} ; \tau=\Omega t ; \\
\bar{K}(\tau)=\frac{C_{1}(t)+i C_{2}(t)}{v \Omega \ell / h^{2}} ; M=\sqrt{\frac{\sigma}{\mu}} B_{0} h ; \alpha=\frac{w_{0}}{\sqrt{\Omega v}} ; \\
R=\frac{\Omega h^{2}}{v} ; V_{x}=\frac{U_{x}}{\Omega \ell} ; V_{y}=\frac{U_{y}}{\Omega \ell} ; k=\frac{n}{\Omega},
\end{array}\right\}
$$

where $\bar{F}(\zeta, \tau)$ is the dimensionless complex translational velocity, $\zeta$ the dimensionless vertical distance; $\tau$ the dimensionless time; $\bar{K}(\tau)$ a function that depends on $\tau$; $M$ the Hartmann number; $\alpha$ the suction/injection velocity parameter; $R$ the Reynolds number; $V_{x}$ and $V_{y}$ the dimensionless velocity amplitudes of oscillation in the $x$ - and $y$-directions; $k$ the ratio of the frequency of oscillation to the angular velocity of the disks.

For the periodic motion in the fluid, it is reasonable to suggest a solution of the form:

$$
\begin{aligned}
& \bar{F}(\zeta, \tau)=\bar{F}_{0}(\zeta)+\bar{F}_{1}(\zeta) \cos k \tau+\bar{F}_{2}(\zeta) \sin k \tau \\
& \bar{K}(\tau)=\bar{K}_{0}+\bar{K}_{1} \cos k \tau+\bar{K}_{2} \sin k \tau
\end{aligned}
$$

where $\bar{K}_{0}, \bar{K}_{1}$ and $\bar{K}_{2}$ are constants and $\bar{F}_{0}(\zeta), \bar{K}_{0}$ correspond to the case of $n=0$. Substituting Eqs. (20)-(21) into Eq. (14), one obtains:

$$
\begin{aligned}
& \bar{F}_{0}^{\prime \prime}-\alpha \sqrt{R} \bar{F}_{0}^{\prime}-\left(M^{2}+i R\right) \bar{F}_{0}=\bar{K}_{0} ; \\
& \bar{F}_{1}^{\prime \prime}-\alpha \sqrt{R} \bar{F}_{1}^{\prime}-k R \bar{F}_{2}-\left(M^{2}+i R\right) \bar{F}_{1}=\bar{K}_{1} ; \\
& \bar{F}_{2}^{\prime \prime}-\alpha \sqrt{R} \bar{F}_{2}^{\prime}+k R \bar{F}_{1}-\left(M^{2}+i R\right) \bar{F}_{2}=\bar{K}_{2},
\end{aligned}
$$




$$
\left.\begin{array}{l}
\bar{F}_{0}(1)=1 ; \bar{F}_{0}(-1)=-1 ; \bar{F}_{0}(0)=0 ; \bar{F}_{1}(1)=0 ; \\
\bar{F}_{1}(-1)=0 ; \bar{F}_{1}(0)=0 ; \bar{F}_{2}(1)=V_{x}+i V_{y} ; \\
\bar{F}_{2}(-1)=-\left(V_{x}+i V_{y}\right) ; \bar{F}_{2}(0)=0
\end{array}\right\}
$$

It should be noted that $\hat{f}(z)$ and $\hat{g}(z)$ are obtained by the solution of Eq. (22).

The dimensionless shear stress components $\bar{T}_{x z}$ and $\bar{T}_{y z}$ in the fluid are found in the complex form as follows:

$$
\begin{aligned}
& \bar{T}_{x z}(\zeta, \tau)+i \bar{T}_{y z}(\zeta, \tau)=A(\zeta)+ \\
& +\frac{V_{y}-i V_{x}}{2}[D(\zeta)-B(\zeta)] \cos k \tau+ \\
& +\frac{V_{x}+i V_{y}}{2}[D(\zeta)+B(\zeta)] \sin k \tau,
\end{aligned}
$$

where

$$
\begin{aligned}
& \bar{T}_{x z}(\zeta, \tau)=\frac{T_{x z}(z, t)}{\mu \Omega \ell / h} ; \bar{T}_{y z}(\zeta, \tau)=\frac{T_{y z}(z, t)}{\mu \Omega \ell / h} ; \\
& A(\zeta)=P_{0} \exp (a \zeta)+Q_{0} \exp (b \zeta) ; \\
& P_{0}=\frac{(1-\cosh b) a}{\sinh a-\sinh b-\sinh (a-b)} ; \\
& Q_{0}=\frac{(\cosh a-1) b}{\sinh a-\sinh b-\sinh (a-b)} ; \\
& a=0.5\left[\alpha \sqrt{R}+\sqrt{\alpha^{2} R+4\left(M^{2}+i R\right)}\right] ; \\
& b=0.5\left[\alpha \sqrt{R}-\sqrt{\alpha^{2} R+4\left(M^{2}+i R\right)}\right] ; \\
& B(\zeta)=P_{1} \exp (c \zeta)+Q_{1} \exp (d \zeta) ; \\
& P_{1}=\frac{(1-\cosh d) c}{\sinh c-\sinh d-\sinh (c-d)} ; \\
& Q_{1}=\frac{(\cosh c-1) d}{\sinh c-\sinh d-\sinh (c-d)} ; \\
& c=0.5\left[\alpha \sqrt{R}+\sqrt{\alpha^{2} R+4\left(M^{2}+i R(1-k)\right)}\right] ; \\
& d=0.5\left[\alpha \sqrt{R}-\sqrt{\alpha^{2} R+4\left(M^{2}+i R(1-k)\right)}\right] ; \\
& D(\zeta)=P_{2} \exp (e \zeta)+Q_{2} \exp (m \zeta) ; \\
& P_{2}=\frac{(1-\cosh m) e}{\sinh e-\sinh m-\sinh (e-m)} ; \\
& Q_{2}=\frac{(\cosh e-1) m}{\sinh e-\sinh m-\sinh (e-m)} ; \\
& e=0.5\left[\alpha \sqrt{R}+\sqrt{\alpha^{2} R+4\left(M^{2}+i R(1+k)\right)}\right] ; \\
& \left.m=0.5\left[\alpha \sqrt{R}-\sqrt{\alpha^{2} R+4\left(M^{2}+i R(1+k)\right)}\right] .\right]
\end{aligned}
$$

It should be emphasized that the above results are valid for all values of $k .\left(\bar{T}_{x z}\right)_{\zeta= \pm 1}$ and $\left(\bar{T}_{y z}\right)_{\zeta= \pm 1}$ correspond to the $x$ - and $y$ - components of the dimensionless force per unit area exerted by the top and bottom disks on the fluid, respectively. The variations of these force components with the Hartmann number $(M)$ and the ratio of the frequency of oscillation to the angular velocity of the disks $(k)$ in the periodic time interval are examined with the help of Figs. 2-7. The solid and dotted lines show the variations on the top and bottom disks, respectively. The examination is made for three different cases when the frequency of oscillation is less than, equal to, and larger than the angular velocity of the disks. Figs. 2-3, Figs. 4-5, and Figs. 6-7 are drawn for $k=0.5, k=1$ and $k=1.25$, respectively.

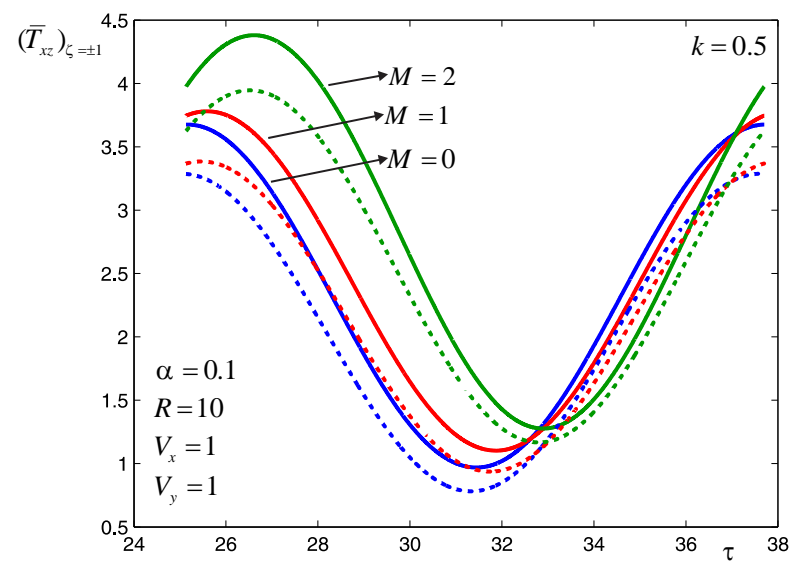

Fig. 2 Variation of $\left(\bar{T}_{x z}\right)_{\zeta= \pm 1}$ with $\tau$ for $k=0.5$ and $M=0,1,2\left(\alpha=0.1, R=10, V_{x}=1, V_{y}=1\right)$

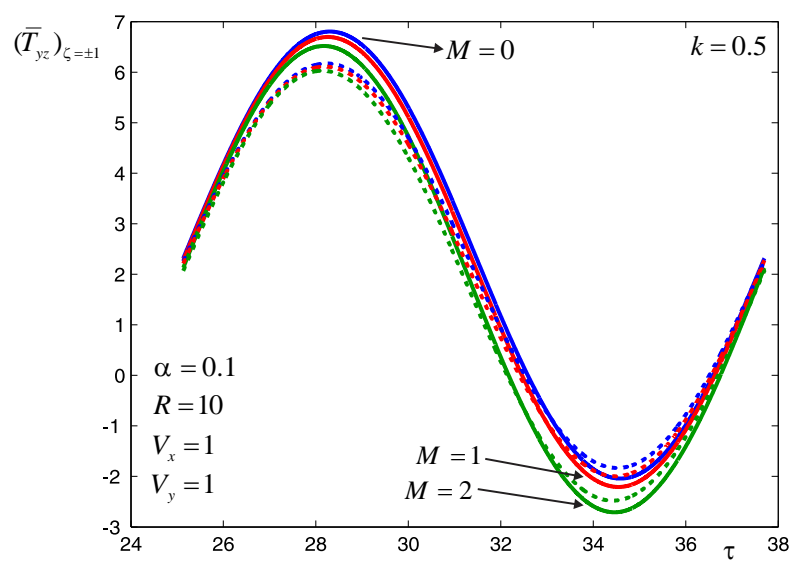

Fig. 3 Variation of $\left(\bar{T}_{y z}\right)_{\zeta= \pm 1}$ with $\tau$ for $k=0.5$ and $M=0,1,2\left(\alpha=0.1, R=10, V_{x}=1, V_{y}=1\right)$

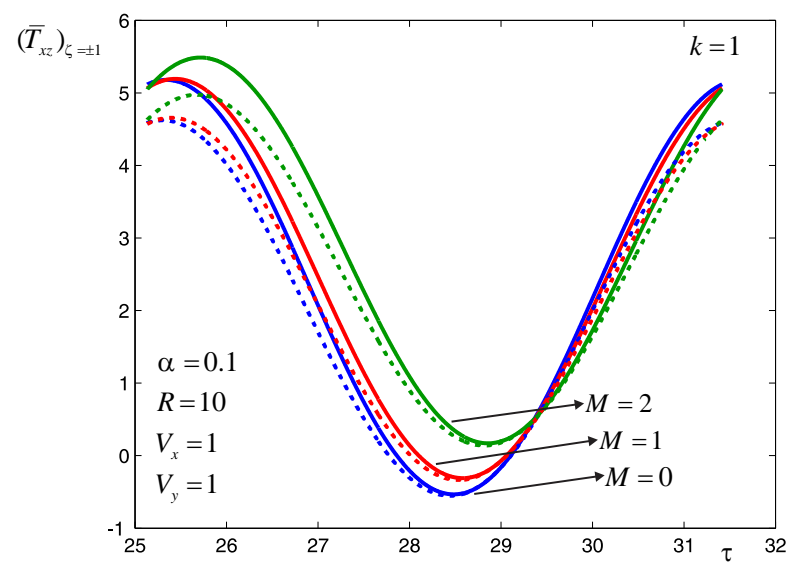

Fig. 4 Variation of $\left(\bar{T}_{x z}\right)_{\zeta= \pm 1}$ with $\tau$ for $k=1$ and $M=0,1,2$ $\left(\alpha=0.1, R=10, V_{x}=1, V_{y}=1\right)$ 


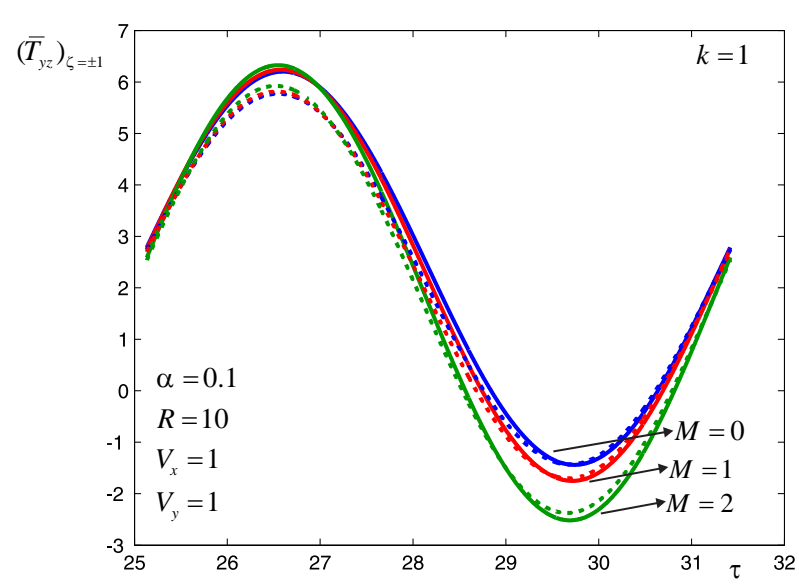

Fig. 5 Variation of $\left(\bar{T}_{y z}\right)_{\zeta= \pm 1}$ with $\tau$ for $k=1$ and $M=0,1,2$ $\left(\alpha=0.1, R=10, V_{x}=1, V_{y}=1\right)$

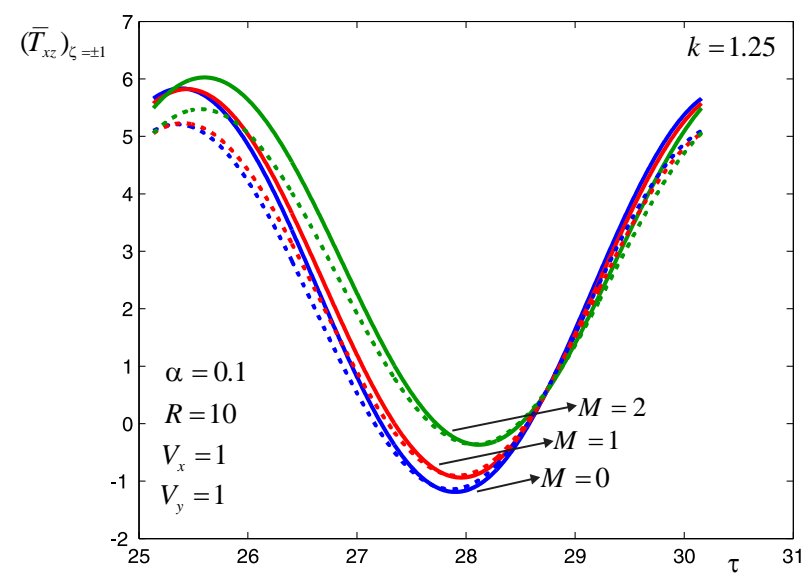

Fig. 6 Variation of $\left(\bar{T}_{x z}\right)_{\zeta= \pm 1}$ with $\tau$ for $k=1.25$ and $M=0,1,2\left(\alpha=0.1, R=10, V_{x}=1, V_{y}=1\right)$

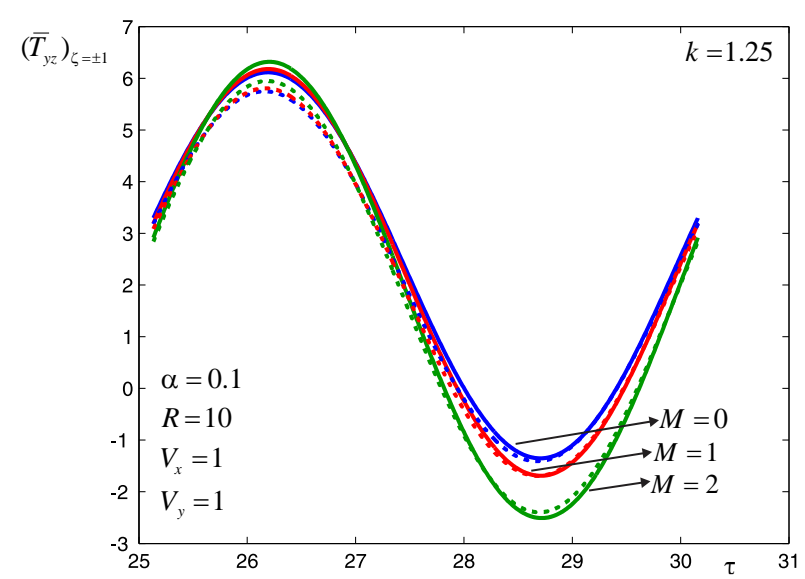

Fig. 7 Variation of $\left(\bar{T}_{y z}\right)_{\zeta= \pm 1}$ with $\tau$ for $k=1.25$ and

$$
M=0,1,2\left(\alpha=0.1, R=10, V_{x}=1, V_{y}=1\right)
$$

\section{Results and discussion}

The calculation of the tangential force on the disks rotating with the same angular velocity about distinct axes is important to determine the material moduli of non-Newtonian fluids. Although the horizontal force on the top disk is the same as that on the bottom disk, they are not equal to each other when the disks are porous. The application of suction or injection is often used for the control of the boundary layers on the disks. In a similar manner, the use of a magnetic field is rather efficient in modifying the boundary layer.

In this paper, the periodic flow due to non-torsional oscillations of eccentric rotating porous disks with the application of a uniform magnetic field is examined. Since the aim of the paper is to extend the analysis in [9] to the magnetohydrodynamic flow, the attention is focused on the effect of the magnetic field. It is worth noting that the results in [9] can be recovered as a special case for $M=0$. It should also be pointed out that the direction of the force exerted by the disk is opposite to that exerted by the fluid. The effects of $\alpha, R, V_{x}$ and $V_{y}$ in the absence of a magnetic field (see [9]) are similar to those in the presence of a magnetic field. The increase of the suction/injection velocity parameter that is based on the axial velocity of fluid leads to the increase and decrease of the horizontal force on the top and bottom disks, respectively. When $R, V_{x}$ and $V_{y}$ increase, the horizontal forces get larger on both the disks.

When the oscillation motion is set up, the flow contains transients for small times. After the initial transients decayed, the motion of the fluid starts to be periodic in time. The examination is made after $\tau=8 \pi$ (see [8,9]) at which the flow already attains its periodic state.

\section{Conclusions}

The major conclusions are summarized as follows:

- The largest values of the $x$-component of the force in the periodic time interval increase with the increase of the Hartmann number.

- The $x$-component of the force on the top disk is greater than that on the bottom disk for small values of the ratio of the frequency of oscillation to the angular velocity of the disks. An opposite result is observed for a small time interval when $k$ increases, but this result is insignificant.

- The direction of the $x$ - component of the force changes within a small time interval when the ratio of the frequency of oscillation to the angular velocity of the disks increases. However, the change of the direction is prevented with the increase of the Hartmann number.

- When the ratio of the frequency of oscillation to the angular velocity of the disks increases, the difference between the $x$ - component of the force on the top disk and that on the bottom disk decreases for any time. A similar effect is also observed in the case of the $y$ - component of the force.

- The variation range of the $y$ - component of the force becomes larger when the Hartmann number increases.

- The variation in the $y$-component is much less in comparison with that in the $x$ - component for every value of the ratio of the frequency of oscillation to the angular velocity of the disks.

\section{Acknowledgements}

The author would like to express his sincere thanks to the referees for their valuable comments and suggestions.

\section{References}

1. Maxwell, B.; Chartoff, R.P. 1965. Studies of a polymer melt in an orthogonal rheometer, Transactions of The Society of Rheology 9(1): 41-52. 
http://dx.doi.org/10.1122/1.548979.

2. Abbott, T.N.G.; Walters, K. 1970. Rheometrical flow systems - part 2: theory for the orthogonal rheometer, including an exact solution of the Navier-Stokes equations, Journal of Fluid Mechanics 40(1): 205-213. http://dx.doi.org/10.1017/S0022112070000125.

3. Rajagopal, K.R. 1982. On the flow of a simple fluid in an orthogonal rheometer, Archive for Rational Mechanics and Analysis 79(1): 39-47. http://dx.doi.org/10.1007/BF02416565. http://link.springer.com/article/10.1007\%2FBF02416565.

4. Rajagopal, K.R. 1992. Flow of viscoelastic fluids between rotating disks, Theoretical and Computational Fluid Dynamics 3(4): 185-206. http://dx.doi.org/10.1007/BF00417912. http://link.springer.com/article/10.1007/BF00417912.

5. Srinivasa, A.R. 2000. Flow characteristics of a multiconfigurational, shear thinning viscoelastic fluid with particular reference to the orthogonal rheometer, Theoretical and Computational Fluid Dynamics 13(5): 305325.

http://dx.doi.org/10.1007/s001620050002.

http://link.springer.com/article/10.1007/s001620050002.

6. Erdoğan, M.E. 1999. Flow due to parallel disks rotating about non-coincident axis with one of them oscillating in its plane, International Journal of Non-Linear Mechanics 34(6): 1019-1030. http://dx.doi.org/10.1016/S0020-7462(98)00072-9. http://www.sciencedirect.com/science/article/pii/S0020746298000729.

7. Erdoğan, M.E. 2000. Unsteady flow between two eccentric rotating disks executing non-torsional oscillations, International Journal of Non-Linear Mechanics 35(4): 691-699.

http://dx.doi.org/10.1016/S0020-7462(99)00051-7. http://www.sciencedirect.com/science/article/pii/S0020746299000517

8. Ersoy, H.V. 2012. Unsteady flow produced by oscillations of eccentric rotating disks, Mathematical Problems in Engineering 2012: 1-14. http://dx.doi.org/10.1155/2012/734784.

http://www.hindawi.com/journals/mpe/2012/734784/.

9. Ersoy, H.V. 2015. Periodic flow due to oscillations of eccentric rotating porous disks, Advances in Mechanical Engineering 7(8): 1-8. http://dx.doi.org/10.1177/1687814015599727. http://ade.sagepub.com/content/7/8/1687814015599727.full.

H. Volkan Ersoy

\section{PERIODIC FLOW DUE TO NON-TORSIONAL OSCILLATIONS OF ECCENTRIC ROTATING POROUS DISKS IN THE PRESENCE OF A MAGNETIC FIELD}

S u m m a r y

This paper deals with the periodic flow induced by non-torsional oscillations of two insulated porous disks while they are initially rotating with the same angular velocity about distinct axes under the application of a magnetic field. An exact solution corresponding to the horizontal force per unit area exerted by the fluid on the top and bottom disks, which is governed by the Hartmann number $(M)$, the suction/injection velocity parameter $(\alpha)$, the Reynolds number $(R)$, the dimensionless velocity amplitude of oscillation in the $x$ - and $y$-directions $\left(V_{x}, V_{y}\right)$, the ratio of the frequency of oscillation to the angular velocity of the disks $(k)$ and the dimensionless time $(\tau)$, is obtained. When the Hartmann number increases, it is found that the largest values of the $x$-component of the force acting on both the top and bottom disks in the periodic time interval increase, and the variation range of the $y$-component of the force becomes wider.

Keywords: Newtonian fluid, magnetohydrodynamics, eccentric rotating porous disks, non-torsional oscillation, periodic flow.

Received February 19, 2016

Accepted June 08, 2017 